sufficiently apart to allow free and easy rolling movement of the paper, when mounted onto the tubular part of the rail. The second idea is simpler since it consists in merely unfolding a cotton sheet lengthwise then tucking the middle six inches together with one hand, placing this on the supra pubic area of the already prone patient and covering her limbs from groin to toes with the two ends of the sheet. This gives maximum cover with minimal embarrassment to the patient and as much exposure and space as necessary for cervical smears when the knees are flexed.

I hope your readers will find my ideas practical. I am etc.

\section{Medical Centre,}

Soest,

British Forces Post Office 106.

1 December 1982

\section{Book Reviews}

Haemorrhoids Cure and Prevention. R L Holt. Abacus Press, Tunbridge Wells, 1980. Pp ix +182 . Hardback $£ 6.50$, Paperback $£ 3.95$.

This book is written by a sufferer from piles who wishes to tell other patients what he has learned about the subject. It would probably be of interest to a sufferer from this often distressing condition. It could be thoroughly recommended except for a eulogy of cryosurgery which comes very close to advertisement.

R SCOTT

A Short Textbook of Surgery. Selwyn Taylor and Leonard Cotton. Hodder \& Stoughton, Sevenoaks. 1982. Pp $1+603$. $£ 7.45$. Illustrated.

This book for medical students can be recommended as concise and well written. It contains all that a London medical student would be expected to know of surgery and is inexpensive.

Qualified doctors who seek to know more may find information in the excellent selection of references, but would probably prefer to have one of the larger standard texts.

R SCOTT

The Pharmacological Basis of Therapeutics. 6th Ed. Alfred Goodman Gilman, Louis $S$ Goodman and Alfred Gilman. Bailliere Tindall, London. 1980. Pp $1+1684$. $£ 28.50$ (Cased.) Illustrated.

If any volume can be said to be the archetypal "standard work" on pharmacology, then this is it. Starting life over 40 years ago when pharmocology was hardly viable as a separate discipline, the latest sixth edition sits monolithically on the shelves of all medical libraries, unchallenged in authority and unsurpassed as a source of reference.

It is old but not aged, having been thoroughly revised and updated by the efforts of a surprising number of 55 contributors. There are many chapters which are entirely new. This is not a work which relies for its authority on its all-inclusiveness, far from it. Rather is it sensibly selective, placing emphasis on recent innovations as well as prototypical drugs, and being unafraid of entering areas of healthy controversy. For example, many drugs are considered which are still under development but which hold promise for the future. There are excellent presentations of the problems and applications of pharmacokinetics, and drug interactions, and the section on toxicology can genuinely be described as modern.

The new chapter on "Neurohumoral Transmission" is welcome and detailed but-just to show that no single volume can be all things to all people-I found the devotion of less than a page to the exciting and fastdeveloping endogenous peptide neurotransmitters disappointing.

A whole section is devoted to "Autacoids", a term devised by Sir Edward Shäfer in 1916 as a substitute for Starling's word "hormone" which is an etymologically unsatisfactory name for certain substances which have inhibitory functions. The revived term "autacoid" embraces a number of substances with differing structure and pharmacological activities but which have in common a natural occurrence within the body-such as histamine, serotonin, certain polypeptides (angiotensin, bradykinin, kallidin), the prostaglandins-and none of which can be classified as neurohumors or true hormones. The fact that their functions and physiological significance is largely unknown is refreshingly taken as a challenge to assemble them together and to discuss? the numerous possibilities they present for therapeutic intervention.

For the Army Medical Officer with an interest in NBC this book offers one particular bonus. Many of us have struggled with the pharmacology and toxicology of the organophosphate anticholinesterases and their potential use as nerve agents in chemical warfare. Pages 100 to 116 present the most lucid exposition of this whole question that $I$ have yet seen.

Others will no doubt find their own particular gems. But no-one who has access to this book will want for background information on virtually the whole range of modern pharmocology and therapeutics.

D ROBERTS

Focus on Vision. R A Weale. Hodder \& Stoughton, Sevenoaks. 1982. Pp $1+$ 178. f5.75. Illustrated.

In the past two decades, ocular physiology has seen advances of far more than merely ophthalmological significance and it is a matter for concern that so much of this germinal material should remain buried and inaccessible to the general reader. Even in medical circles, regrettably, the import, for instance, of the new knowledge on the developmental aspects of the human visual system, seems often to have escaped attention. This knowledge is not esoteric and it is not merely of academic interest; it is of high practical moment and its application can, and does, have profound human implications.

Obviously, no general book of the type under review can consist wholly of new material and Professor Weale's eminently readable 'Focus on Vision' does not pretend to do so. But what the author does tell us of long-established embryological, anatomical and physiological material, is presented from the stand-point of a mathematically sophisticated biological engineer with a keen awareness of the importance of generalisation. This is not to imply that the text is difficult to follow. Professor Weale has a notable talent for clear, interesting and humorous exposition and the reader will find 
himself drawn painlessly along surprising new pathways of mental perception.

Probably the most important parts of this excellently produced and illustrated book, are those concerned with recent development in the knowledge of the receptive fields of the retinal elements and their cortical correspondences and, in particular, the effect upon the functional maturation of these, of early stimulus deprivation. The names of Hubel and Wiesel will certainly take rank in the future with those of Helmholz, Pavlov and Sherrington and it is important that their work should be widely known and understood. The essence of it is contained herein. Professor Weale's book is divided arbitrarily into four sections but, inevitably, there is a good deal of overlap. Within these four sections, he deals with classical and recent ocular and neuro-ophthalmological physiology; gives us an unusual study of 'light as a governor' in which he treats of the nature of a stimulus and the effects, both on the eye and the whole body of light periodicity in the context of circadian rhythms; provides us with an elegant and easily followed account of the embryology of the eye; a valuable dissertation on neonatal ocular physiology and development; an interesting study of the eye in old age and a remarkably complete study of the nature of visual perception. Within its stated limits, it is an excellent primer for the general reader and quite indispensable for the FRCS (0) candidate. There is a splendid bibliography and a good index and the book is produced on first-class paper in sections actually sewn together. All this makes the price of $£ 5.75$ a bargain indeed.

\section{R M YOUNGSON}

The Beecham Manual for Family Practice. Dr J Fry. MTP Press Ltd. Lancaster. 1982. Pp $3+227$. £11.95. Illustrated.

This new edition is a revision which has compressed what was in 3 volumes previously into a reasonably sized single book. The writing and diagrams are well spaced, clear, and of a very high standard, so nothing has been lost. The book does not claim to cover the whole extent of Family Practice and of necessity in its format tends to be dogmatic. There could thus be some argument about criteria for home management of e.g. myocardial infarct and long term management of urine tract infection. These are of minimal importance and the reviewer was greatly impressed by what it contained. It will be of particular value to younger GPs and the Primary Health Team but its clear guidance with many common problems in General Practice will make it attractive to all those involved in Primary
Care.

It will require regular updating but could become a favourite in GP Libraries.

W G KILPATRICK

Schistosomiasis-Epidemiology, Treatment and Control. $P$ Jordan and G Webbe. William Heinemann Medical Books Ltd. London. 1982. Pp $1+345$. f19.00. Illus-
trated.

Next to malaria schistosomiasis is probably the most important of all tropical infectious diseases. The three main component species, S. haematobium, S. mansoni and $S$. japonicum cause untold human misery and enormous economic burdens for poor countries. This very readable compact book-the successor to Human
Schistosomiasis by the same authors published in 1969 - summarises most of what need usefully be know about the disease. The authors have successfully cof laborated with a team of other experts to describe th\$ ancient disease. Epidemiology and control, parasite life cycles and the clinical presentation and pathology ape all fully covered. There are also helpful chapters on the latest diagnostic laboratory techniques including currem developments in serological diagnosis. Good account are given of the effective newer schistocidal drugs. A chapters are adequately illustrated. References are ex tensive and up to date. This is an important book for all involved with schistosomiasis. It is especially reconf mended as a vade mecum for the field worker at the front line in the endless war against this importaon disease. By present day standards it is modestly priced at just under $£ 20$.

J B STEWART

The Physical Examination -An Atlas for General Prae tice. L Lodewick and A D G Gunn. MTP Press Lt Lancaster. 1982. Pp $3+$ 271. £19.95. Illustrated.

"This book is intended for medical students and general practitioners and is an excellently produced and well illustrated atlas of methods of examining ahe patient. The reviewer would have liked the descriptions of tests for motor function and reflexes to have iffes cated the nerve roots tested and the photograph of the ophthalmoscopic examination does not suggest that the examiner must use his $\mathbf{R}$ eye for examining the patieft $\mathrm{c}_{\mathrm{g}}$ $\mathbf{R}$ eye and vice versa. These are minor criticisms i⿱ book which would be a helpful aid to medical studant and reference for general practitioners."

W G KILPATRIEK

Atlas of Ophthalmology. M G Glasspool. MTP press Ltd. Lancaster. 1982. Pp $7+$ 121. £17.50. Illustra原禹

In 128 pages, $\mathrm{Mr}$ Glasspool has succeeded eminentl well in covering, and, in most cases, effectively illustrap ing, all the common ophthalmic conditions likely to bo encountered by the general practitioner. But this bood is more than simply an atlas of eye photographs; contains excellently clear and succinct accounts of the gross anatomy and elementary physiology of the eye, useful outline of examination methods, a section on the refractive errors and brief accounts of the eye io systemic disease and of neuro-ophthalmology. The sec. tion on squint is especially well done and very clearl $\bar{x}$. illustrates the important method of diagnosis by cove test.

The text is, of necessity, synoptic, but is none the worse for that. It is entirely orthodox and may be taken as currently authoritative. Almost all controversia开 matters are avoided and proper emphasis is given to at? matters of importance.

Ophthalmology must needs tolerate but a mino share of the time allocated to a medical education. IT is thus of great importance that the student should select a text which will not burden him with a mase of indigestible information, but which, at the same time, will effectively present the essentials of the subo ject. It seems to me that $\mathrm{Mr}$ Glasspool's atlas does. exactly that. I would recommend it, unreservedly, tou medical students, to general physicians as a hand $\rho$ reference text and to ophthalmic juniors as a mositu useful introduction to the subject. 